\title{
Simulation von CNFET basierten Digitalschaltungen
}

\author{
O. Soffke, P. Zipf, M. Velten, and M. Glesner \\ FG Mikroelektronische Syteme, TU Darmstadt, Germany
}

\begin{abstract}
Zusammenfassung. Einwandige Kohlenstoff Nanoröhrchen können sowohl halbleitende als auch metallische Eigenschaften aufweisen, je nachdem wie die Röhrchenachse im Vergleich zur Anordnung der Kohlenstoffatome verläuft. Dies wird durch den sogenannten Aufrollvektor bestimmt. Halbleitende Nanoröhrchen können für Transistoren (CNFETs) verwendet werden, deren Verhalten sich mit einer modifizierten Version von Berkeley Spice 3f5 simulieren läßt. Die aus diesen Simulationen gewonnenen Parameter werden zur Parametrisierung von SystemC Modellen aus CNFETs bestehender Grundschaltungen verwendet, was zu einer um Größenordnungen höheren Simulationsgeschwindigkeit bei hoher Genauigkeit führt.
\end{abstract}

Abstract. Single walled carbon nanotubes (CNT) can be either metallic or semiconducting depending on the tube's orientation in relation to the configuration of the carbon atoms. This is determined by the so-called chiral vector. Semiconducting CNT can be used in transistors (CNFET) which can be simulated by a modified version of Berkeley Spice $3 f 5$. The parameters determined by these simulations are used to parameterise SystemC models of some basic building blocks yielding fast simulations with high accuracy.

\section{Einleitung}

Einwandige Kohlenstoff Nanoröhrchen stellen ein vielversprechendes Material bei der Suche nach den Bauelementen der Zukunft dar. Man kann sich diese Röhrchen dadurch entstanden denken, dass eine Graphenschicht, also eine Graphitschicht mit der Dicke einer Atomlage, zu einem Zylinder aufgerollt wird.

Correspondence to: O. Soffke

(soffke@mes.tu-darmstadt.de)
Eine Eigenschaft von Kohlenstoff Nanoröhrchen ist, dass diese entweder halbleitend oder metallisch sein können, je nachdem wie die Röhrchenachse im Vergleich zur Anordnung der Kohlenstoffatome verläuft. Dies wird durch den sogenannten Aufroll- oder Chiralvektor bestimmt. Aus theoretischen berlegungen weiß man, dass bei rein zufällig gewähltem Aufrollvektor ein Drittel der entstehenden Röhrchen metallisch und zwei Drittel halbleitend sind. Zur Verwendung in Transistoren sind natürlich nur die halbleitenden Nanoröhrchen von Interesse.

Der restliche Beitrag ist wie folgt gegliedert: In Abschnitt 2 werden die physikalischen Eigenschaften von Kohlenstoff-Nanoröhrchen vorgestellt und Abschnitt 3 beschäftigt sich mit der Simulation von auf diesen Elementen basierenden Transistoren mit Spice. Schließlich wird die Abstraktion der Eigenschaften in Abschnitt 4 diskutiert und die Ergebnisse in Abschnitt 5 zusammengefasst.

\section{Physikalische Eigenschaften von Kohlenstoff - Nanoröhrchen}

\subsection{Geometrische Eigenschaften}

Kohlenstoff-Nanoröhrchen kann man sich durch Aufrollen eines Streifens vorstellen, der aus einer Graphenschicht herausgeschnitten ist. Die Orientierung dieses Streifens relativ zur Anordnung der beteiligten Kohlenstoffatome wird durch den Aufrollvektor $\boldsymbol{c}$ bestimmt. Dieser lässt sich durch eine Linearkombination der in Abb. 1 definierten Gittervektoren $\boldsymbol{a}_{1}$ und $\boldsymbol{a}_{2}$ darstellen:

$\boldsymbol{c}=n_{1} \boldsymbol{a}_{1}+n_{2} \boldsymbol{a}_{2} ; n_{1}, n_{2} \in \mathbb{Z}$.

Die Symmetrielinie durch die C-Atome wird als Armchairund die durch die Mittelpunkte der Sechsecke als ZigzagLinie bezeichnet. Demzufolge nennt man Nanoröhrchen, deren Aufrollvektor entlang einer solchen Symmerielinie

Published by Copernicus GmbH on behalf of the URSI Landesausschuss in der Bundesrepublik Deutschland e.V. 


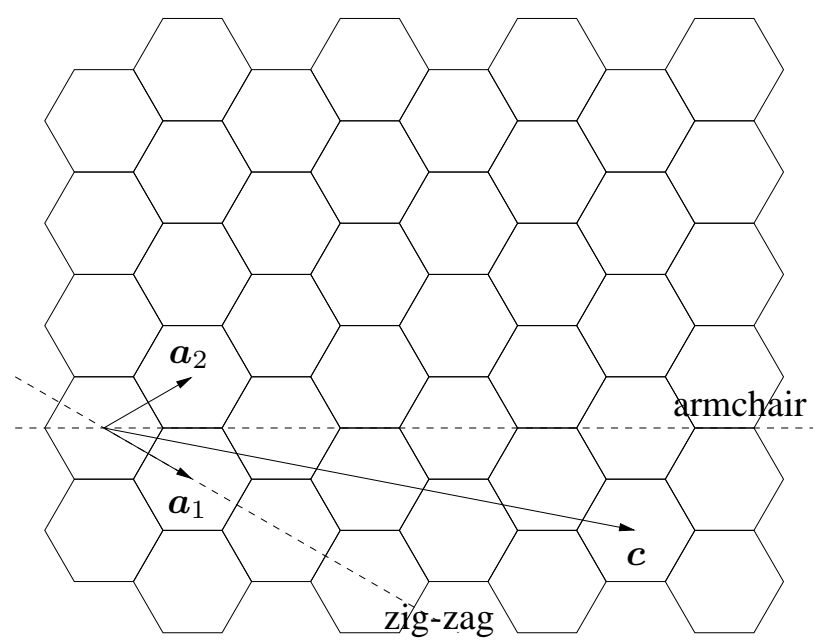

Abbildung 1. Gittervektoren $\boldsymbol{a}_{1}$ und $\boldsymbol{a}_{2}$ sowie Aufrollvektor $\boldsymbol{c}$ eines Kohlenstoff-Nanoröhrchens mit $n_{1}=4$ und $n_{2}=2$.

verläuft, entsprechend Armchair- bzw. Zigzag-Röhrchen. Aus Symmetriegründen ist es ausreichend Aufrollvektor zu betrachten, die zwischen diesen beiden Linien verlaufen. Für diese Vektoren gilt:

$0 \leq n_{2} \leq n_{1}$

Der Umfang des Kohlenstoff-Nanoröhrchens ist schließlich durch die Länge des Aufrollvektors gegeben:

$$
\begin{aligned}
|\boldsymbol{c}| & =\sqrt{\left(n_{1} \boldsymbol{a}_{1}+n_{2} \boldsymbol{a}_{2}\right)^{2}} \\
& =\sqrt{n_{1}^{2}\left(\boldsymbol{a}_{1}\right)^{2}+2 n_{1} n_{2} \boldsymbol{a}_{1} \boldsymbol{a}_{2}+n_{2}^{2}\left(\boldsymbol{a}_{2}\right)^{2}} .
\end{aligned}
$$

Mit der Definition $a_{0}:=\left|\boldsymbol{a}_{1}\right|=\left|\boldsymbol{a}_{2}\right|$ gilt für die auftretenden Skalarprodukte:

$$
\begin{aligned}
& \left(\boldsymbol{a}_{1}\right)^{2}=a_{0}^{2} \\
& \left(\boldsymbol{a}_{2}\right)^{2}=a_{0}^{2} \\
& \boldsymbol{a}_{1} \boldsymbol{a}_{2}=\frac{a_{0}^{2}}{2} .
\end{aligned}
$$

Damit folgt für den Umfang des Nanoröhrchens:

$$
|\boldsymbol{c}|=a_{0} \sqrt{n_{1}^{2}+n_{2}^{2}+n_{1} n_{2}} .
$$

Darüber hinaus besitzt das Kohlenstoff-Nanoröhrchen eine Verschiebungssymmetrie, d.h. die Struktur entlang der Röhrchenachse wiederholt sich periodisch. Die Periode wird durch den Vektor $\boldsymbol{t}$ beschrieben, was in Abb. 2 dargestellt ist. In diesem Zusammenhang bezeichnet man eine Periode als 1-D Einheitszelle (Yang, 1999). Auch der Vektor $\boldsymbol{t}$ läßt sich als Linearkombination der Gittervektoren darstellen:

$\boldsymbol{t}=t_{1} \boldsymbol{a}_{1}+t_{2} \boldsymbol{a}_{2} ; t_{1}, t_{2} \in \mathbb{Z}$

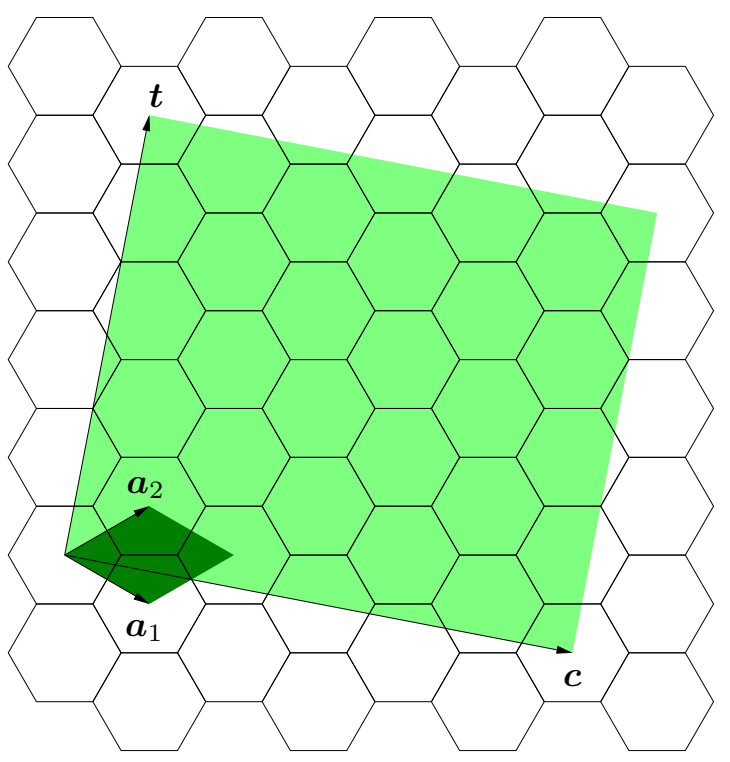

Abbildung 2. 1-D Einheitszelle eines Kohlenstoff-Nanoröhrchens mit $n_{1}=4$ und $n_{2}=2$.

Es läßt sich zeigen, dass für die beiden Koeffizienten $t_{1}$ und $t_{2}$ gilt:

$$
\begin{aligned}
t_{1} & =-\left(n_{1}+2 n_{2}\right) / g \\
t_{2} & =\left(2 n_{1}+n_{2}\right) / g \\
\text { mit } \quad g & =\operatorname{GGT}\left(2 n_{1}+n_{2}, n_{1}+2 n_{2}\right),
\end{aligned}
$$

wobei GGT(.) die Bestimmung des Größten Gemeinsamen Teilers bedeutet. Weitere Informationen dazu finden sich in Shukla and Bahar (2004).

Im dargestellen Beispiel gilt $n_{1}=4$ und $n_{2}=2$. Damit folgt für

$$
\begin{aligned}
g & =\operatorname{GGT}(10,8)=2 \\
t_{1} & =-(4+2 \cdot 2) / 2=-4 \quad \text { und } \\
t_{2} & =(2 \cdot 4+2) / 2=5 .
\end{aligned}
$$

Für die Länge der 1-D Einheitszelle und damit für die „Periodendauer" ergibt sich:

$$
|\boldsymbol{t}|=\frac{\sqrt{3} a_{0}}{g} \sqrt{n_{1}^{2}+n_{2}^{2}+n_{1} n_{2}} .
$$

\subsection{Elektrische Eigenschaften}

Für die elektrischen Eigenschaften von KohlenstoffNanoröhrchen ist, wie in allen Materialien, die Bandstruktur verantwortlich.

Die Wellenfunktion der Elektronen muß dabei in Umfangrichtung $2 \pi$-periodisch sein, was zu einer Quantisierung der Komponente $k_{c}$ des Wellenvektors in Umfangsrichtung führt (Maultzsch, 2004):

$k_{c}=\frac{2 \pi q}{|c|}, q \in \mathbb{Z}$ 


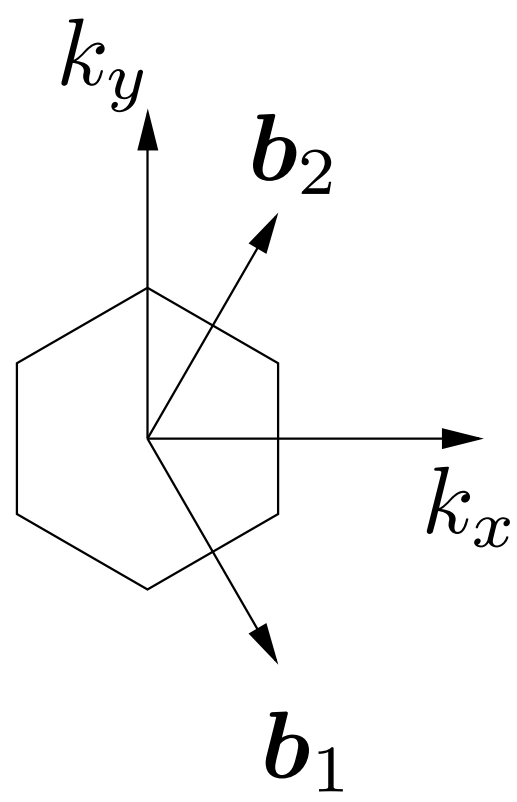

Abbildung 3. Erste Brillouin Zone von Graphen und Basisvektoren des reziproken Gitters.

Im Gegensatz dazu ist die axiale Komponente $k_{t}$ kontinuierlich. Dies ergibt die möglichen Wellenvektoren:

$\boldsymbol{k}=k_{c} \boldsymbol{e}_{c}+k_{t} \boldsymbol{e}_{t}=\frac{2 \pi q}{|\boldsymbol{c}|} \boldsymbol{e}_{c}+k_{t} \boldsymbol{e}_{t}$,

wobei $\boldsymbol{e}_{c}$ und $\boldsymbol{e}_{t}$ die Einheitsvektoren in Umfangsrichtung bzw. in Richtung der Röhrchenachse sind. Schließlcih lassen sich diese Wellenvektoren als Geraden in der $\boldsymbol{k}$-Ebene beschreiben, die durch

$\frac{\sqrt{3}\left|\boldsymbol{a}_{i}\right|}{2}\left(n_{1}+n_{2}\right) \cdot k_{x}+\frac{\left|\boldsymbol{a}_{i}\right|}{2}\left(n_{2}-n_{1}\right) \cdot k_{y}=2 \pi q$

gegeben sind. Dabei stellen $k_{x}$ und $k_{y}$ die Komponenten der auftretenden Wellenvektoren in $x$ - bzw. $y$-Richtung dar.

Die zu den entsprechenden Wellenvektoren gehörende Energie läßt sich aus der Dispersionsrelation von Graphen (Saito et al., 1992) ermitteln:

$E(\boldsymbol{k})= \pm t_{0} \sqrt{3+2 \sum_{i=1}^{3} \cos \left(\boldsymbol{k} \boldsymbol{a}_{i}\right)}$

mit $\boldsymbol{a}_{3}=\boldsymbol{a}_{2}-\boldsymbol{a}_{1}$ und $t_{0} \approx 3 \mathrm{eV}$.

Die Gittervektoren $\boldsymbol{b}_{1}$ und $\boldsymbol{b}_{2}$ des reziproken Gitters ergeben sich zu:

$\boldsymbol{b}_{1}=2 \pi \frac{\boldsymbol{a}_{2} \times \boldsymbol{e}_{n}}{\boldsymbol{a}_{1}\left(\boldsymbol{a}_{2} \times \boldsymbol{e}_{n}\right)}, \quad \boldsymbol{b}_{2}=2 \pi \frac{\boldsymbol{e}_{n} \times \boldsymbol{a}_{1}}{\boldsymbol{a}_{2}\left(\boldsymbol{e}_{n} \times \boldsymbol{a}_{1}\right)}$,

wobei $\boldsymbol{e}_{n}$ den Normalenvektor auf die Gitterebene darstellt. Daraus lassen sich die Ecken der 1. Brillouin Zone (BZ) bestimmen und nachweisen, dass die Energie $E(\boldsymbol{k})$ in diesen

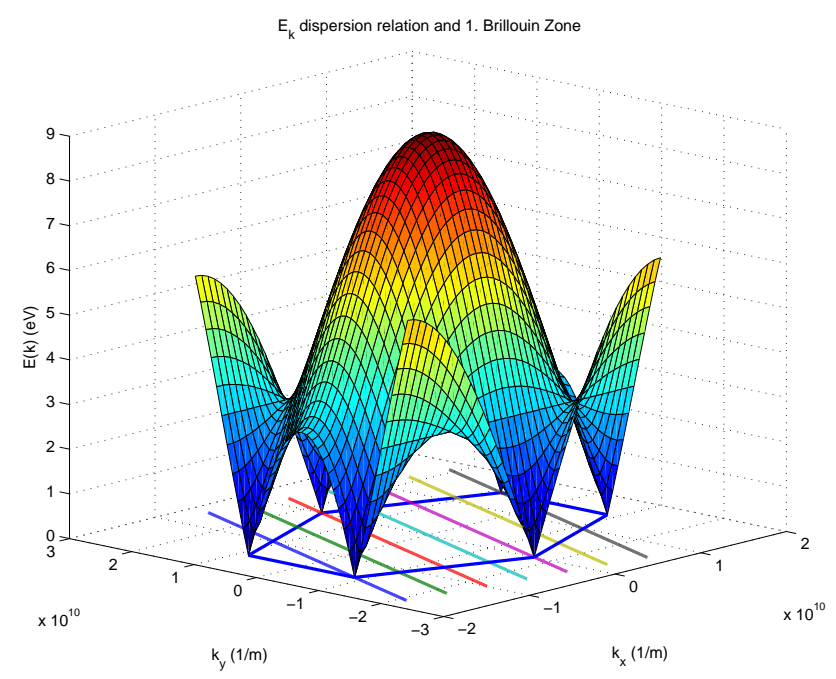

Abbildung 4. $E(\boldsymbol{k})$ Dispersionsrelation für Graphene. Darüber hinaus sind die auftretenden Wellenvektoren in der $\boldsymbol{k}$-Ebene eingezeichnet. Da einige dieser Geraden durch die Ecken der 1. BZ laufen, ist das dargestellte Kohlenstoff-Nanoröhhrchen metallisch.

Ecken zu Null wird. Die Gittervektoren $\boldsymbol{b}_{1}$ und $\boldsymbol{b}_{2}$ des reziproken Gitters sowie die 1. BZ sind in Abb. 3 dargestellt.

In Abb. 4 sieht man schließlich die Dispersionsrelation von Graphen, d.h. die zu den Wellenvektoren $\boldsymbol{k}$ gehörende Energie $E(\boldsymbol{k})$. Zusätzlich sind in der $\boldsymbol{k}$-Ebene die auftretenden Wellenvektoren eingezeichnet. Da die Energie in den Ecken der 1. BZ verschwindet, hat das Nanoröhrchen nur dann keine Bandlücke, wenn eine Ecke der 1. BZ einem möglichen Wellenvektor entspricht (der Energienullpunkt ist hier gleich dem Ferminiveau). Dies ist gleichbedeutend damit, dass eine der Geraden durch eine der Ecken läuft. Durch geometrische Analyse läßt sich zeigen, dass dies nur dann möglich ist, wenn $\left(n_{1}-n_{2}\right) / 3$ ganzzahlig ist (Raja et al., 2004). Dies bedeutet, dass das Kohlenstoff-Nanoröhrchen sich metallisch verhält, wenn diese Bedingung erfüllt ist. Andernfalls ist das Röhrchen halbleitend. Bei zufällig entstehenden Kohlenstoff-Nanoröhrchen sind demzufolge ein Drittel der Röhrchen metallisch und zwei Drittel halbleitend.

Halbleitende Kohlenstoff-Nanoröhrchen kommen für den Aufbau von Transistoren in Frage. Bei diesen Transistoren kann der Strom durch das Röhrchen durch ein elektrisches Feld gesteuert werden, ähnlich wie bei konventionellen MOSFETs. Aufgrund der eindimensionalen Struktur des Nanoröhrchens erfolgt der Transport allerdings nahezu ballistisch (Rahman et al., 2003).

\section{Simulation mit Spice}

Für die Simulation auf Transistorebene kommt das von Dwyer et al. (2004) vorgestellte I-V-SPICE zum Einsatz. Dabei handelt es sich um eine modifizierte Version von Berke- 


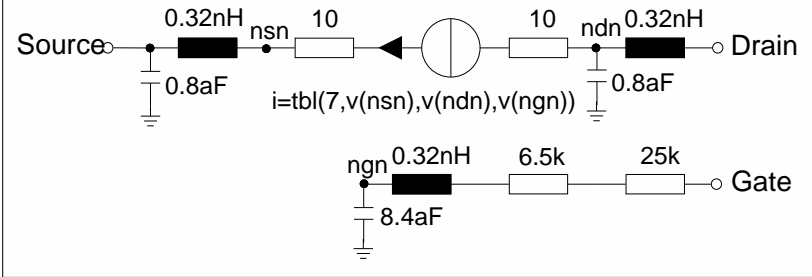

(a)

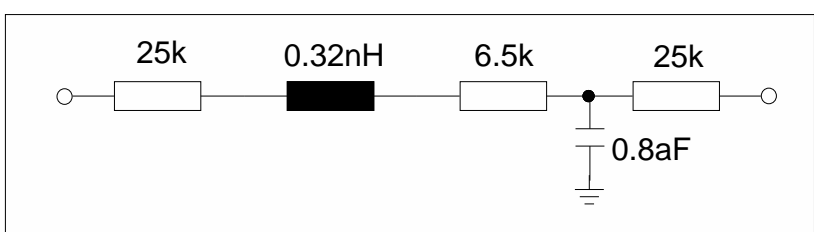

(b)

Abbildung 5. Das von Dwyer et al. (2004) verwendete Modell eines CNFET (a) und eines metallischen Kohlenstoff-Nanoröhrchen (b). Die in (a) verwendete Stromquelle ist durch tabellarisierte Meßwerte realisiert.

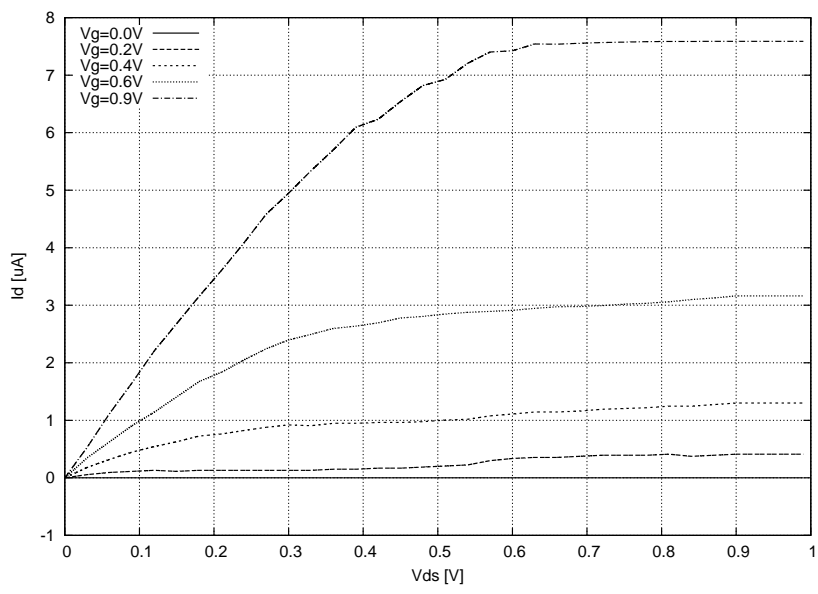

Abbildung 6. Ausgangskennlinienfeld eines n-Typ CNFET.

ley Spice 3f5. Der Quellcode wurde dahingehend verändert, dass Strom-Spannungskennlinien von Bauelementen in tabellarischer Form dem Simulator zugänglich gemacht werden können. Dadurch ist es möglich reale Messdaten von hergestellten CNFETs für die Simulation nanoelektronischer Schaltungen zu verwenden. Zusätzlich ergänzten Dwyer et al. (2004) das Modell um dynamische Effekte in Form von parasitären Induktivitäten und Kapazitäten. Ein Modell von metallischen Kohlenstoff Nanoröhrchen wurde ebenfalls vorgestellt. Die in I-V-SPICE verwendeten Modelle für CNFETs und metallische Kohlenstoff-Nanoröhrchen sind in Abb. 5 dargestellt. Das Ausgangskennlinienfeld eines n-Typ CNFET zeigt Abb. 6. Diese Kurven repräsentieren im Wesentlichen das Verhalten der Stromquelle aus Abb. 5a.

Im Rahmen dieses Beitrags wurden sowohl CNFETs als auch metallische Kohlenstoff Nanoröhrchen verwendet, um ein NAND Gatter zu implementieren. Mit diesem NAND Gatter wurde anschließend ein flankengetriggertes D-FlipFlop aufgebaut. Sowohl das Gatter wie auch das FlipFlop wurden durch ausführliche Simulationen in I-V-Spice charakterisiert.

\section{Abstraktion mit SystemC}

Für den Entwurf komplexer digitaler Schaltungen werden abstrahierte Modelle benötigt, die eine höhere Simulationsgeschwindigkeit als durch Spice erlauben. Daher wurden die durch Spice-Simulationen ermittelten Daten verwendet, um SystemC Modelle des Flip-Flops und des Gatters zu entwickeln.

Die SystemC Abstraktionen modellieren dabei auch Effekte wie eine vergrößerte Durchlaufzeit bei Verletzung der Setup-Zeit des Flip-Flops. Natürlich wird in einem solchen Fall eine entsprechende Warnmeldung generiert. Im Falle des Gatters werden auch Effekte berücksichtigt, die auftreten, wenn beide Eingänge nur für kurze Zeit gleichzeitig auf logisch eins liegen. In einem solchen Fall unterschreitet die Ausgangsspannung in der Spice-Simulation die 50\% Schwelle der Versorgungsspannung nicht. Bei vergleichbarem Timing der Eingangssignale zeigt auch der Ausgang der SystemC Simulation keine Veränderung. Dies ist ein wesentlicher Unterschied zu herkömmlichen abstrakten Modellen, die ein solches Gatter als Boolesche Funktion mit nachgeschaltetem Verzögerungselement modellieren. In diesem Fall würde am Ausgang ein kurzer Glitch auftreten, obwohl dieser in der realen Schaltung nicht vorhanden ist.

Die Abbildungen 7 und 8 vergleichen die Simulationsergenisse von SystemC und Spice miteinander. Die gute bereinstimmung der Ergebnisse bezüglich des Zeitverhaltens ist deutlich zu erkennen. In Abb. 7 sieht man darüber hinaus die erwähnte „Filtereigenschaft“ des NAND Gatters, und dass sich diese auch in der SystemC Simulation wiederfindet.

Im Falle des Flip-Flops wurde der Zeitpunkt der Änderung des Dateneingangs mit dem Zyklus näher an die Taktflanke geschoben. Man erkennt deutlich, dass sich die bei einer Verletzung der Setup-Zeit auftretende Verzögerung in der SystemC Simulation sehr gut widerspiegelt. Bei einer ,größeren“ Verletzung ,kippt" der Ausgang schließlich nicht mehr. Auch dieses Verhalten zeigt die SystemC Simulation. In Zipf et al. (2005) findet sich eine detaillierte Beschreibung der SystemC Implementierung. 


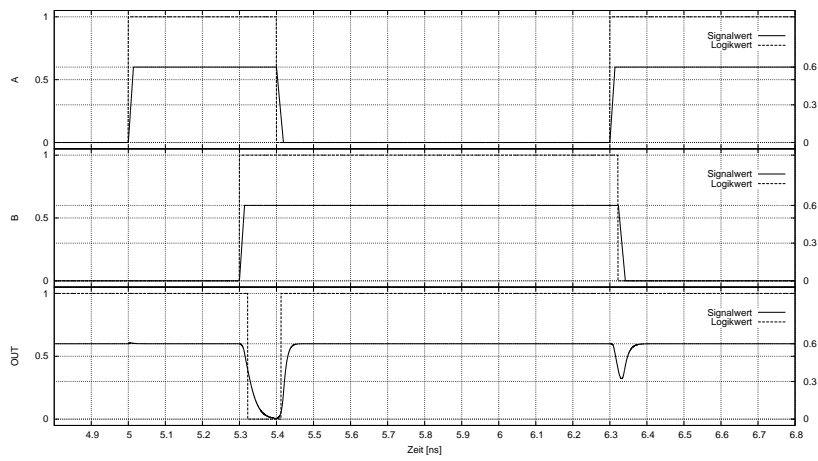

Abbildung 7. Vergleich des Zeitverhaltens des Flip-Flops bei Simulation mit SystemC und Spice.

\section{Zusammenfassung}

In diesem Beitrag werden die geomtrischen, physikalischen und elektrischen Eigenschaften von Kohlenstoff-Nanoröhrchen reflektiert. Diese Nanoröhrchen können entweder metallische oder halbleitende Eigenschaften aufweisen, wobei sich die halbleitenden zum Einsatz in Transistoren eignen. Eine modifizierte Version von Spice kann zur Simulation dieser Transistoren bzw. einfacher Grundschaltungen verwendet werden. Die so ermittelten Parameter dienen zur Konfiguration von SystemC Modellen, die Simulationen erlauben, die fast genauso akkurat aber um Größenordnungen schneller sind als entsprechende mit Spice.

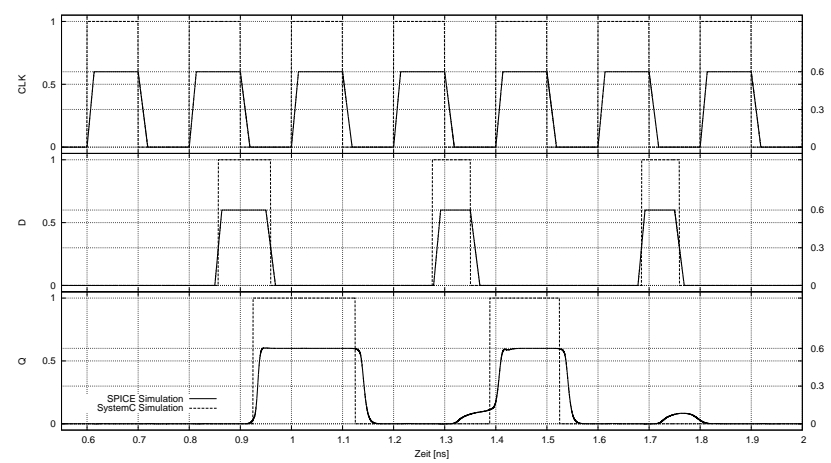

Abbildung 8. Vergleich des Zeitverhaltens des NAND Gatters bei Simulation mit SystemC und Spice.

\section{Literatur}

Dwyer, C., Cheung, M., and Sorin, D.: Semi-empirical SPICE Models for Carbon Nanotube FET Logic, 4th IEEE Conference on Nanotechnology, pp. 386-388, 2004.

Maultzsch, J.: Vibrational properties of carbon nanotubes and graphite, Ph.D. thesis, Technische Universität Berlin, 2004.

Rahman, A., Guo, J., Datta, S., and Lundstrom, M. S.: Theory of Ballistic Nanotransistors, IEEE Transactions on Electron Devices, 50, 1853-1864, 2003.

Raja, T., Agrawal, V. D., and Bushnell, M. L.: A Tutorial on the Emerging Nanotechnology Devices, in: Proc. of the 17th Intern. Conf. on VLSI Design (VLSID’04), 2004.

Saito, R., Fujita, M., Dresselhaus, G., and Dresselhaus, M. S.: Electronic structure of chiral graphene tubules, Appl. Phys. Lett., 60, 2204-2206, 1992.

Shukla, S. K. and Bahar, R. I., eds.: Nano, Quantum and Molekular Computing, Kluwer, 2004.

Yang, L.: Band-gap cahnge of carbon nanotubes: Effect of small uniaxial and torsional strain, Phsical Review B, 60, 13 874-13 878, 1999.

Zipf, P., Soffke, O., Velten, M., and Glesner, M.: Abstrakte Modellierung der Eigenschaften von nanoelektronischen CNTElementen in SystemC, in: Workshop Entwurfsmethoden für Nanometer VLSI Design, Informatik LIVE 2005, Bonn, 2005. 